\title{
Influence of Experimental Hyperglycemia on Microvascular Blood Perfusion of Pancreatic Islet Isografts
}

\author{
Michael D. Menger, * Peter Vajkoczy, * Rosmarie Leiderer, * Sabine Jäger, ${ }^{\star}$ and Konrad Messmer * \\ *Institute for Surgical Research, University of Munich, 8000 Munich, Federal Republic of Germany; and ${ }^{\ddagger}$ Department of General Surgery, \\ University of Saarland, 6650 Homburg, Saar, Federal Republic of Germany
}

\section{Abstract}

The influence of hyperglycemia on the microvascular blood perfusion of pancreatic islet isografts of Syrian golden hamsters was analyzed by direct visualization of the islet's microvasculature by means of in vivo fluorescence microscopy. The experiments were performed using the hamster dorsal skinfold preparation, which allows for quantitative analysis of the microcirculation of islets grafted on the striated skin muscle. Islets were isolated from inbred hamsters by collagenase digestion and subsequently transplanted in normoglycemic (controls; $n=8)$ and hyperglycemic $(65 \mathrm{mg} / \mathrm{kg}$ streptozotocin intravenously; $\boldsymbol{n}=10$ ) recipients. In both groups, revascularization of the islet grafts was completed on day 10 after transplantation. Quantitative analysis of capillary blood perfusion on days 6,10 , and 14 revealed no differences in functional capillary density and capillary red blood cell velocity of islets grafted into normoglycemic as compared to hyperglycemic animals. However, islet capillaries were significantly wider in hyperglycemic recipients $(11.9 \pm 1.3 \mu \mathrm{m}, P<0.01)$ as compared to normoglycemic controls $(8.9 \pm 0.4 \mu \mathrm{m})$. The increase of capillary diameters resulted in a significant rise $(P<0.01)$ of mean capillary blood perfusion from $1.76 \pm 0.39 \mathrm{nl} / \mathrm{min}$ in controls to $2.88 \pm 0.63 \mathrm{nl} / \mathrm{min}$ in hyperglycemic recipients, indicating an increase in microvascular blood perfusion due to hyperglycemia. From these results it is concluded that hyperglycemia is associated with higher capillary blood perfusion in revascularized islet isografts, similarly as known for pancreatic islets in situ. (J. Clin. Invest. 1992.90:1361-1369.) Key words: intravital fluorescence microscopy - quantitative microcirculatory analysis • hamster • revascularization • islet microvasculature

\section{Introduction}

A variety of studies have demonstrated that transplantation of isolated pancreatic islets has the potential to reverse experimental diabetes (1). However, although survival and function of the grafts depend primarily on their nutritional blood supply, little is known about the microvascular blood perfusion of those freely transplanted islets. Furthermore, the influence of hyperglycemia on the microvasculature of grafted islets re-

Address correspondence to Dr. M. D. Menger, Institute for Surgical Research, University of Munich, Marchioninistrasse 15, 8000 München 70, Federal Republic of Germany. 1992.

Received for publication 13 May 1991 and in revised form 2 March

J. Clin. Invest.

(c) The American Society for Clinical Investigation, Inc.

0021-9738/92/10/1361/09 \$2.00

Volume 90, October 1992, 1361-1369 mains controversal. It is well known that hyperglycemia increases microvascular blood perfusion in islets of Langerhans in situ (2-5). However, in 1987 Sandler and Jansson (6) reported on reduced blood flow to islet grafts, when autotransplanted beneath the kidney capsule of hyperglycemic recipients, and the authors suggested that hyperglycemia may adversely affect the process of vascularization of islet grafts. In contrast, intravital microscopic studies from our laboratory revealed that angiogenesis and revascularization of single islet isografts transplanted onto striated muscle is not affected by hyperglycemia (7).

In 1989 Jansson and Sandler (8) elaborated further the influence of hyperglycemia on blood perfusion of autotransplanted pancreatic islets in diabetic rats. Although the mechanism underlying the impairment of graft perfusion during continuous hyperglycemia remained unexplained, it has been suggested that this phenomenon may not only reflect an effect on the process of revascularization, but on the intrinsic regulation of blood perfusion of the graft (8).

With the aim of clarifying these contradictory results, we have analyzed in vivo the influence of hyperglycemia on capillary blood perfusion of islet isografts during the process of revascularization as well as over a time period after revascularization was completed. For this purpose we used the hamster dorsal skinfold preparation, which consists of striated muscle and skin and allows for free transplantation of isolated pancreatic islets (9). The technique enables for repetitive and quantitative analysis of microcirculatory parameters by means of intravital fluorescence microscopy in the awake animal for a prolonged period of time.

\section{Methods}

Animals. Inbred Syrian golden hamsters, 6-8-wk old and $60-80 \mathrm{~g}$ body wt, were used for microcirculatory studies; islet isolation was performed from pancreatic glands of 12 -wk-old animals ( $120 \mathrm{~g}$ body $\mathrm{wt}$ ). The animals were housed one per cage and had free access to tap water and standard pellet food (Altromin; Lage, Federal Republic of Germany) throughout the experiment.

Islet isolation. For isolation of pancreatic islets we used a modified collagenase digestion technique (10). Syrian golden hamsters were anesthetized with $50 \mathrm{mg} / \mathrm{kg}$ sodium pentobarbital (Nembutal; Abbott Laboratories, North Chicago, IL) intraperitoneally. After midline laparotomy, the pancreas was perfused with $3 \mathrm{mg}$ of neutral red (Sigma Chemical Co., St. Louis, MO) dissolved in $20 \mathrm{ml}$ of Hanks' solution (H6136; Sigma Chemical Co.) via the abdominal aorta, staining the pancreatic tissue slightly red. Subsequently, the pancreas was gently dissected free, and removed. The excised gland was cut into $\sim 30-40$ pieces and put in a sterilized glass vial containing $20 \mathrm{mg}$ of collagenase from Clostridium histolyticum (Serva Feinbiochemica GmbH \& Co., Heidelberg, FRG) dissolved in $5 \mathrm{ml}$ of Hanks' solution. The vial was rapidly shaken at a temperature of $37^{\circ} \mathrm{C}$ for $10-15 \mathrm{~min}$ until the tissue had disintegrated. The digest was sedimented three times in ice cold Hanks' solution, and the isolated islets were handpicked for free trans- 
plantation, using a stereomicroscope at a magnification of 24 . By this procedure we were constantly able to pick $600-800$ islets from one donor pancreas. Macromorphological integrity of the islets, i.e., exclusion of fragments, was controlled photomicroscopically at a magnification of 64.20 islets of each sample were used to verify their viability by means of dithizone staining (11). Microscopic handpicking procedure guaranteed single exocrine free islets for transplantation.

Islet transplantation. As the transplantation site we used the hamster dorsal skinfold chamber, which contains striated muscle and skin, and allows for in vivo observation of the microvasculature by means of intravital fluorescence microscopy in the awake animal. As demonstrated in previous studies $(9,12)$, the skinfold preparation is a valid model for free pancreatic islet transplantation, in which the striated skin muscle served as a bed for the transplants. The chamber and its implantation have been described by Endrich et al. $(13,14)$ in detail. Briefly, under pentobarbital anesthesia $(50 \mathrm{mg} / \mathrm{kg}$ body wt intraperitoneally; Abbott Laboratories), the animals were fitted with two symmetrical titanium frames, positioned on the dorsal skinfold, sandwiching the extended double layer of skin. One layer of skin, subcutaneous tissue, and skin muscle, as well as both layers of the musculus retractor were completely removed in a circular area of $\sim 15 \mathrm{~mm}$ in diameter, and the remaining layers (consisting of striated skin muscle, subcutaneous tissue, and skin) were covered with a removable cover slip, which was incorporated into one of the titanium frames (Fig. 1). The chamber was implanted two days before islet transplantation, allowing the microcirculation of the host tissue to recover from surgery and anesthesia. Only skinfold preparations presenting without signs of inflammation and with normal microcirculation, as reported earlier (13), were allowed for the experiments. The animals tolerated the chambers well and showed no signs of discomfort. In particular, no effect on sleeping and feeding habits were observed. Neither implantation of the chamber nor administration of the fluorescent tracer for intravital microscopy affected macrohemodynamic parameters such as mean arterial blood pressure and heart rate.

\begin{tabular}{lll}
\hline titanium frame & observation window \\
\hline skin & \\
\hline subcutaneous tissue & cover slip \\
\hline skin muscle & \\
\hline m. retractor & \\
\hline skin muscle & \\
\hline subcutaneous tissue & \\
\hline skin & \\
\hline
\end{tabular}

Figure 1. Schematic cross-sectional diagram of the hamster dorsal skinfold and the arrangement of the chamber. Two symmetrical titanium frames are positioned on the dorsal skinfold of the hamster, so as to sandwich the extended double layer of skin. One layer of skin, subcutaneous tissue, and skin muscle, as well as both layers of the musculus retractor are completely removed in a circular area of $\sim 15$ $\mathrm{mm}$ in diameter, and the remaining layers (consisting of skin muscle, subcutaneous tissue, and skin) are covered with a removable cover slip, which is incorporated into one of the titanium frames. Using intravital fluorescence microscopy the microcirculation of the striated skin muscle and subcutaneous tissue visualized through the observation window of the chamber can be analyzed quantitatively in the awake animal over a prolonged period of time.
Subsequently, after isolation, $\sim 8-10$ islets, evenly distributed in size (150-200 $\mu \mathrm{m})$ were transplanted into each skinfold chamber. During the transplantation procedure the cover slip of the chamber was removed and under microscopic control the islets were placed on the striated muscle tissue within the chamber. This procedure guarantees that islets are transplanted as single grafts, allowing assessment of capillary blood perfusion of individual islets.

Intravital fluorescence microscopy. For in vivo microscopic observation, the awake animals were immobilized in a Plexiglass tube, and the skinfold preparation, containing the islet grafts, was attached to the microscope stage. The stage was placed on a computer-controlled microscope desk, which allows for repeated scanning of each individual islet for intravital microscopy. After intravenous application of $0.2 \mathrm{ml}$ 5\% FITC-labeled dextran $\left(M_{\mathrm{r}} 150,000\right.$; Sigma Chemical Co., St. Louis, MO) in vivo microscopy was performed using a modified Orthoplan microscope (Leitz, Wetzlar, FRG) with a $75 \mathrm{~W}, \mathrm{XBO}$, xenon lamp attached to a Ploemo-Pak illuminator with an $I_{2}$ blue filter block (Leitz) for epi-illumination. The observations were recorded by means of a silicon-intensified-target video camera (COHU 4400; Prospective Measurements, San Diego, CA) and transferred to a video system for off-line evaluation.

Microvascular blood perfusion is characterized by the density of the microvessels, the red blood cell velocity, and the vessel diameters. All three parameters were analyzed quantitatively by in vivo microscopy. The density of the microvessels was assessed for each individual islet. For analysis of capillary red blood cell velocity and capillary diameters a vertical line was drawn on the video screen. The vessels crossing this line were randomly chosen for analysis. Functional capillary density (length of all red blood cell perfused capillaries per islet area) was determined by means of the Computer Assisted Microcirculation Analysis System (CAMAS; Zeintl, Neckargmünd, Federal Republic of Germany), including planimetric area measurement for the assessment of the size of individual islets. Capillary red blood cell velocity was measured in the centerline of the microvessels using the dual slit technique (Velocity Tracker model 102B; I.P.M. Inc., San Diego, CA). Vessel diameters were analyzed by means of image shearing (Image Shearing Monitor model 907; I.P.M. Inc.).

Functional capillary density was analyzed using a $\times 20$ long distance working objective ( numerical aperture 0.32; Leitz, Wetzlar, Federal Republic of Germany) achieving a total magnification at the video system of 450 (screen diagonal: $330 \mathrm{~mm}$ ). Capillary red blood cell velocity and capillary diameters were evaluated using a $\times 32$ long distance working objective (numerical aperture 0.40; Leitz), with a total magnification of 720 . In addition, individual capillary blood perfusion (CBP) was calculated from the capillary red blood cell velocity (CRBCV) and capillary diameter (CD) for each capillary as:

$$
\mathrm{CBP}=\pi(\mathrm{CD} / 2)^{2} \times \mathrm{CRBCV} / \mathrm{K}
$$

where $\mathrm{K}$ represents the Baker/Wayland factor (15) considering the parabolic velocity profile of blood in microvessels. According to $\mathrm{Li}$ powsky and Zweifach (16) a factor of 1.3 was used for microvessels with diameters of $\sim 10 \mu \mathrm{m}$.

In addition, the size of the microvascular network was analyzed by measuring the total diameter of the area encompassed by the islet's microvessels.

Experimental groups. Four days before islet transplantation, hyperglycemia was induced in 10 animals by intravenous application of streptozotocin ( $65 \mathrm{mg} / \mathrm{kg}$ body wt; Sigma Chemical Co.). All streptozotocin-injected animals were hyperglycemic at the time of islet transplantation, presenting with nonfasting serum glucose concentrations above $18 \mathrm{mmol} /$ liter (photometric analyzer PCP-6121; Eppendorf, Hamburg, Federal Republic of Germany). Untreated animals $(n=8)$ with serum glucose levels $<9 \mathrm{mmol} /$ liter served as controls. Quantitative analysis of the microvascular blood perfusion of the islet grafts was performed during the process of revascularization (day 6) and after completion of revascularization (days 10 and 14) in hyperglycemic animals and in normoglycemic controls. 
Histological examinations. Dorsal skinfold preparations of normoglycemic $(n=6)$ and hyperglycemic recipients $(n=6)$, including the islet grafts, were fixed on days 6,10 , and 14 by both vascular perfusion and superfusion with $2.5 \%$ glutaraldehyde in $0.1 \mathrm{M} \mathrm{Na}$-cacodylate buffer ( $\mathrm{pH} \mathrm{7.4)}$ for $30 \mathrm{~min}$. The tissue was then carefully excised and put in the same fresh fixative for another $24 \mathrm{~h}$ at $4^{\circ} \mathrm{C}$, before small pieces, each containing one islet, were dissected under a stereomicroscope. These specimens were then processed for electron microscopy, employing an EM 10A microscope (Car) Zeiss Inc., Thornwood, NY) operated at $60 \mathrm{kV}$

Statistical analyses. Results are expressed as means \pm standard deviation. Statistical analyses include one-way analysis of variance and unpaired Student's $t$ test for comparison between the groups. Paired Student's $t$ test, including Bonferroni probabilities for repeated measurements, were performed for analyzing differences within each group. Differences were considered significant at a $P<0.05$ level.

\section{Results}

General characteristics of hamsters receiving transplants. Streptozotocin-diabetic animals presented with polydipsia, polyuria, and weight loss during the experiment. Body weight of the animals decreased significantly $(P<0.01)$ from $72.1 \pm 9.3 \mathrm{~g}$ before streptozotocin administration to $59.3 \pm 8.8 \mathrm{~g}$ at the time of transplantation, and no further increase was observed on days $6(56.0 \pm 14.7 \mathrm{~g}), 10(57.5 \pm 6.2 \mathrm{~g})$, and 14 $(59.3 \pm 8.3 \mathrm{~g})$ after transplantation. In contrast, normoglycemic animals increased body weight significantly $(P<0.01)$ from $70.9 \pm 5.2 \mathrm{~g}$ to $81.9 \pm 6.4 \mathrm{~g}$ during the $18-\mathrm{d}$ observation period.

In streptozotocin-treated animals nonfasting serum glucose concentration was significantly elevated at the time of transplantation $(20.7 \pm 1.6 \mathrm{mmol} /$ liter $)$, and remained increased throughout the entire experiment, i.e., $20.4 \pm 1.3 \mathrm{mmol} /$ liter on day $6,20.3 \pm 1.1 \mathrm{mmol} /$ liter on day 10 , and $20.0 \pm 1.3 \mathrm{mmol} /$ liter on day 14 after transplantation, indicating that islet transplantation did not reverse hyperglycemia. In nontreated animals serum glucose concentration was found $<9 \mathrm{mmol} /$ liter throughout the entire 14-d observation period.
In awake hyperglycemic animals macrohemodynamic analysis revealed a mean arterial blood pressure of $116.4 \pm 14.1$ $\mathrm{mmHg}$ and heart rate of $340.0 \pm 49.9 \mathrm{~min}^{-1}$ at the time of transplantation and was not found significantly changed after 14 days. Similarly, in normoglycemic controls no changes in mean arterial pressure and heart rate $(121.1 \pm 20.3 \mathrm{mmHg}$ and $348.6 \pm 56.7 \mathrm{~min}^{-1}$ at the time of transplantation) were observed.

In both groups, the microvessels of the islet grafts were free from signs of inflammation, i.e., increase of microvascular permeability (proven by the absence of extravasation of the fluorescent macromolecule FITC-Dextran 150,000) and interstitial edema. In parallel, within the host tissue no inflammationinduced phenomena, such as tortuosity of the microvessels and white blood cell accumulation, were observed.

Revascularization of islet isografts. First signs of revascularization of the islet isografts could be demonstrated on day 2 after transplantation, being characterized as capillary sprouts, originating from the venular segments of capillaries and postcapillary venules of the striated muscle. During the following days protrusion of sprouts was observed, leading to interconnection of individual sprouts and formation of a honeycomblike glomerular network on day 6 after transplantation. The total diameter of this glomerular network as well as the density of the microvessels increased until revascularization of the islet was completed on day 10 (Fig. 2). The morphological structure of this microvascular network is very similar to the microvasculature of pancreatic islets in situ. With regard to the process of revascularization no differences were observed between normoglycemic and hyperglycemic recipients. In normoglycemic animals quantitative analysis of the total diameter of the islet's microvascular network showed a significant increase ( $P$ $<0.05$ ) from $358.5 \pm 28.7 \mu \mathrm{m}$ on day 6 to $411.7 \pm 53.9 \mu \mathrm{m}$ on day 10 after transplantation without further change on day 14 . In hyperglycemic animals the diameter values were found comparable to those of normoglycemic controls, i.e., $375.6 \pm 33.3$

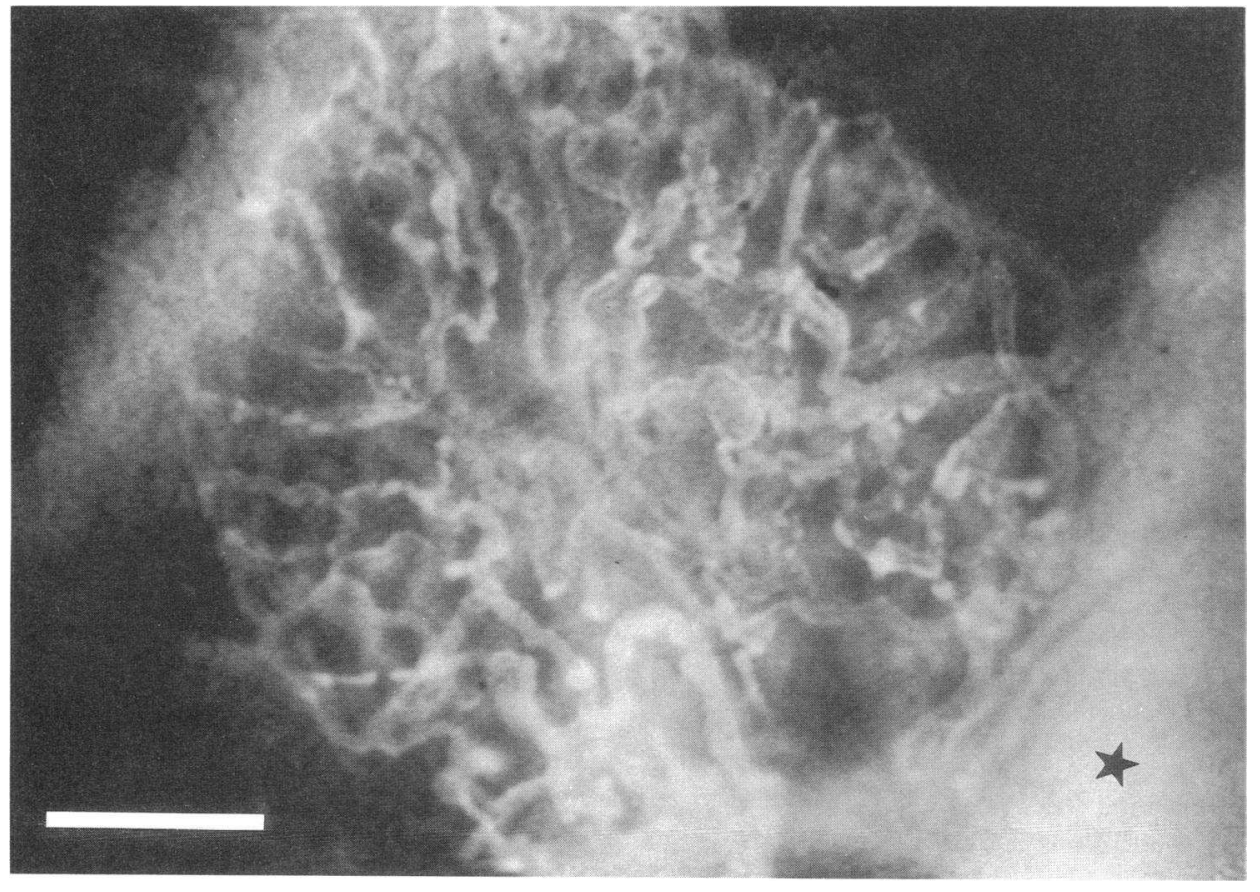

Figure 2. Microvasculature of a pancreatic islet isograft. Microvascular network of a pancreatic islet isograft 10 days after free transplantation onto striated muscle within the chamber preparation of the Syrian golden hamster, located adjacent to a postcapillary venule $(\star)$ of the muscle microcirculation. Note the glomerulum-like network of capillaries, indicating complete revascularization. Intravital fluorescence microscopy, contrast enhancement with 5\% FITC-Dextran 150,000 intravenously; bar represents $100 \mu \mathrm{m}$. 
$\mu \mathrm{m}$ on day 6 and $383.2 \pm 29.5 \mu \mathrm{m}$ on day 10 after transplantation.

Microvascular blood perfusion of islet isografts. Microvascular blood perfusion was analyzed in 65 single islet isografts of 8 normoglycemic, and in 76 islet grafts of 10 hyperglycemic recipients. Functional capillary density of the islets increased significantly in normoglycemic as well as in hyperglycemic animals from day 6 to day 10 after transplantation, and no difference was observed between streptozotocin-treated animals and controls (Fig. 3). In both groups, no further increase in capillary density could be demonstrated on day 14 after transplantation, indicating that revascularization has been completed within the first 10 days, and density of perfused capillaries in islet grafts of hyperglycemic animals was not different from that of normoglycemic recipients (Fig. 3).

Within the islet isografts red blood cell velocity and diameters were analyzed of 388 capillaries in normoglycemic, and of 471 capillaries in hyperglycemic recipients. Analysis of capillary red blood cell velocity revealed a significant increase $(P$ $<0.01$ ) from $0.36 \pm 0.08$ and $0.38 \pm 0.07 \mathrm{~mm} / \mathrm{s}$ at day 6 to $0.54 \pm 0.05$ and $0.57 \pm 0.09 \mathrm{~mm} / \mathrm{s}$ at day 10 after transplantation in both, normoglycemic and hyperglycemic animals. Comparison between the two groups did not show significant differences, neither on day 6 nor on day 10 after transplantation. On day 14 the values of capillary red blood cell velocity were not different from those measured on day 10 (Fig. 3).

In normoglycemic recipients the diameters of the islet's capillaries were found reduced significantly $(P<0.05)$ from day 6 to day 10 after transplantation, amounting to $\sim 9 \mu \mathrm{m}$, while no further changes were encountered on day 14 (Fig. 4). In hyperglycemic recipients capillary diameters were found signifcantly higher as compared to those of islets growing in normoglycemic controls (Fig. 5). The diameters of these microvessels did not decrease after revascularization was completed, but remained wider $(\sim 11.5 \mu \mathrm{m})$ as compared to normoglycemic controls $(P<0.01)$ throughout the entire observation period (Fig. 4).

Calculation of individual capillary blood perfusion in islet isografts of normoglycemic recipients revealed no changes dur-

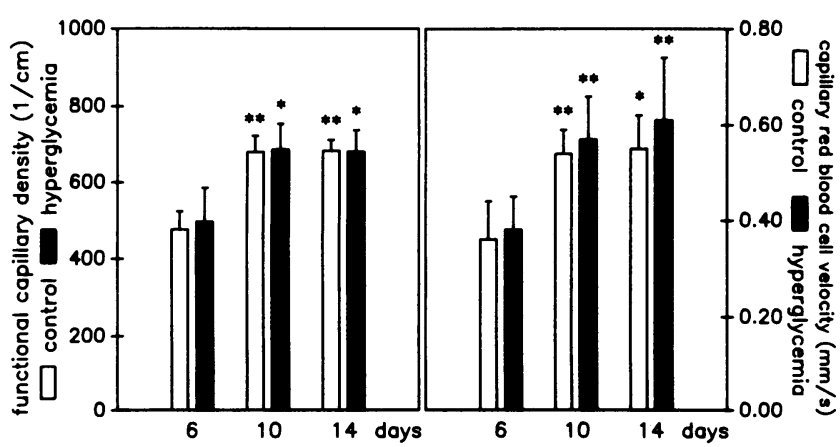

Figure 3. Functional capillary density and capillary red blood cell velocity of islet isografts in normoglycemic and hyperglycemic recipients. Pancreatic islet isografts were transplanted into normoglycemic $(\square, n=8)$ and hyperglycemic $(\square, n=10)$ recipients. Functional capillary density and capillary red blood cell velocity of the islet grafts were analyzed during revascularization (day 6 ) and after completion of revascularization (days 10 and 14) by means of a computer-assisted image analysis system and the dual slit technique. Mean $\pm \mathrm{SD} ;{ }^{*} P$ $<0.05 ;{ }^{* *} P<0.01$ vs. day 6; paired Student's $t$ test (comparison within the groups) including Bonferroni correction for repeated measurements.

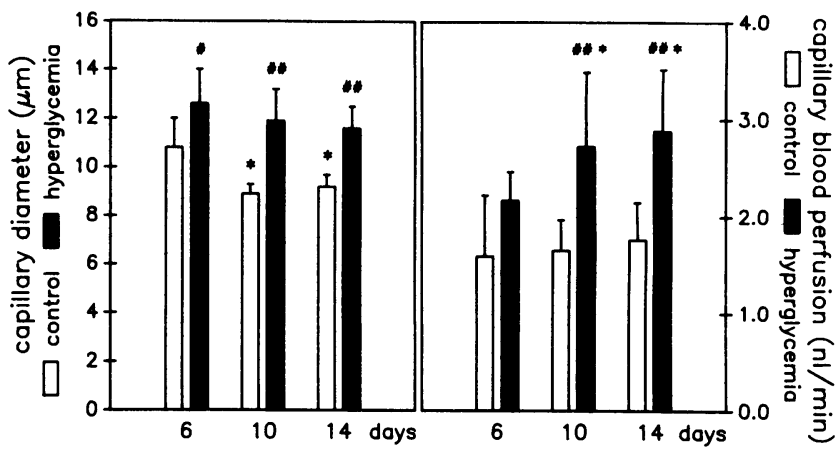

Figure 4. Capillary diameters and capillary blood perfusion of islet isografts in normoglycemic and hyperglycemic recipients. Capillary diameters and capillary blood perfusion of islet isografts transplanted into normoglycemic $(\square, n=8)$ and hyperglycemic $(\square, n=10)$ recipients, analyzed during revascularization (day 6 ) and after completion of revascularization (days 10 and 14). Diameters were measured by means of image shearing; capillary blood perfusion was calculated from the individual diameter and red blood cell velocity. Mean $\pm S D$; ${ }^{*} P<0.05$ vs. day 6; paired Student's $t$ test (comparison within the groups) including Bonferroni correction for repeated measurements; ${ }^{\text {" }} P<0.05$; $P<0.01$; unpaired Student's $t$ test (comparison between the groups).

ing the process of revascularization (day 6) compared to the time period when revascularization was completed (day 10 and 14, Fig. 4). In contrast, in hyperglycemic recipients capillary blood perfusion was found enhanced $(P<0.05)$ between day 6 and day 10 and 14 after transplantation. In addition, islet capillaries of hyperglycemic animals showed a significantly $(P$ $<0.01$ ) higher blood perfusion on days 10 and 14 as compared to normoglycemic controls.

Transmission electron microscopy of islet isografts. Transmission electron microscopy of the grafts revealed normal ultrastructure of islet cells in both normoglycemic and hyperglycemic recipients (Fig. 6). Comparison of cell nuclei displayed no significant difference between the two groups, indicating the absence of $\beta$-cell hypertrophy in islet grafts of hyperglycemic recipients. However, augmentation of secretory granules and of rough endoplasmatic reticulum was occasionally observed in islet grafts of hyperglycemic recipients (Fig. 6, $B$ and $C$ ), indicating the high metabolic activity of these cells. This notion was confirmed by the apparently great number of free ribosomes and polyribosomes in those cells (Fig. 6, $B$ and $C$ ).

\section{Discussion}

Hyperglycemia and microvascular blood perfusion of islet grafts. Experimental studies on blood perfusion of pancreatic islets in situ have demonstrated an increase of microvascular blood flow as result of hyperglycemia (2-5). Although the underlying mechanism could not be clarified by studies in which microspheres were used for measurement of islet blood flow (4, 5 ), dilatory effects of hyperglycemia on the microvasculature of the islets have been proposed $(2,3)$. Using the fluorescent dye vasoflavine for direct visualization, Hellerström and Hellman (3) demonstrated in hyperglycemic animals wider and more contorted islet capillaries as well as a larger blood cell content within the islet's microvasculature.

In 1987 Sandler and Jansson (6) investigated microvascular blood flow in islet autografts (clusters) after 1 wk $37^{\circ} \mathrm{C}$ culture and transplantation beneath the kidney capsule by 

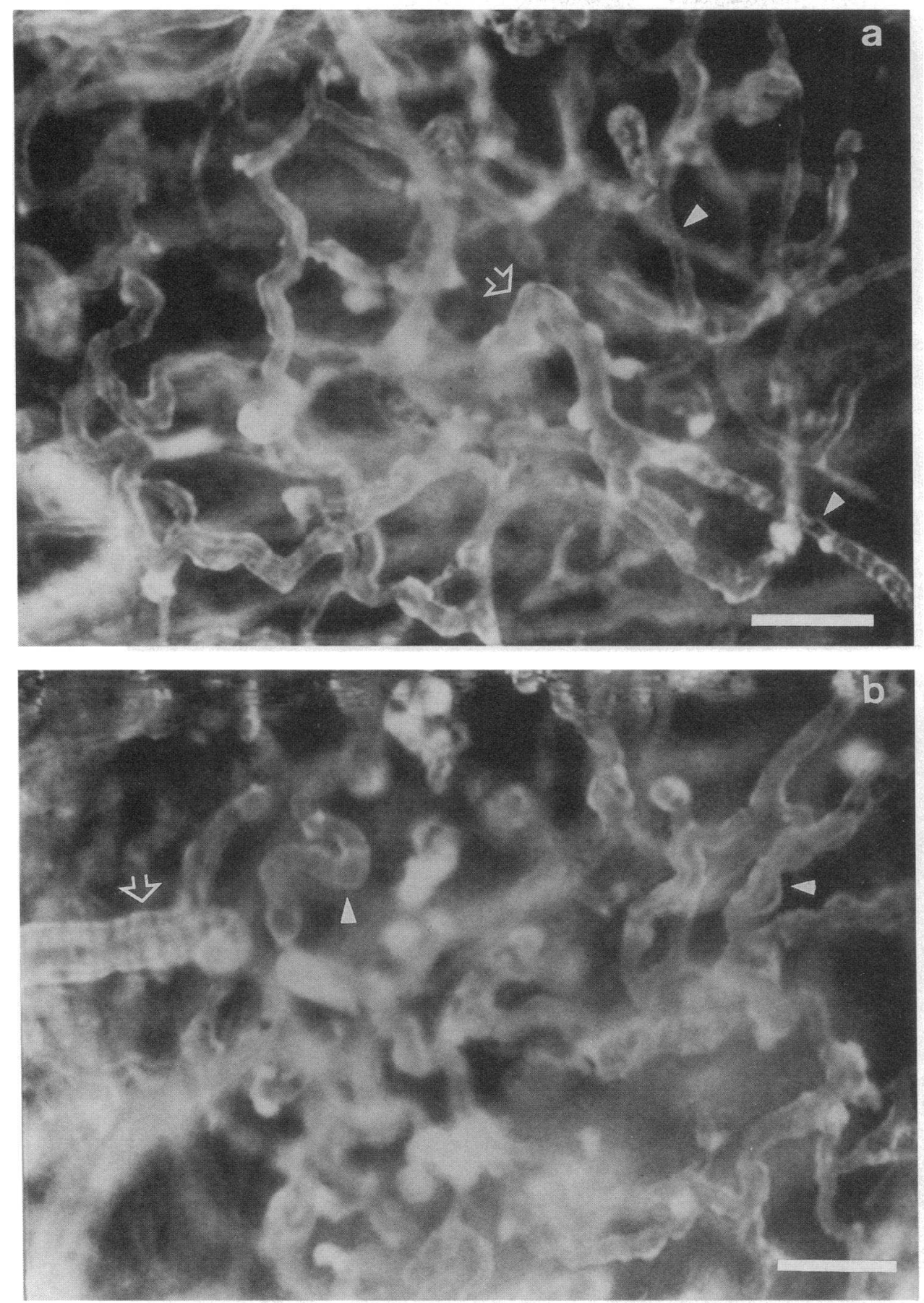

Figure 5. Capillary network of islet isografts in normoglycemic and hyperglycemic recipients. High magnification of the microvascular network of islet isografts 10 days after transplantation onto striated muscle of a normoglycemic recipient ( $a$ ) and of a streptozotocin-induced diabetic recipient $(b)$. In both normoglycemic and hyperglycemic animals, the microvasculature of the islet grafts is characterized by the homogeneous density of the capillaries within the glomerulum-like network. The microvasculature of the islet graft of the hyperglycemic recipient reveals capillary widening and tortuosity $(b ; \triangleright)$ as compared to the microvasculature of the islet graft of the normoglycemic recipient $(a ; \triangleright)$. In addition, note the dilatation of the central feeding microvessel $(\Rightarrow)$ supplying the islet graft of the hyperglycemic recipient $(b)$ as compared to that of the normoglycemic control $(a ; \Rightarrow)$. Intravital fluorescence microscopy, contrast enhancement with 5\% FITC-Dextran 150,000 intravenously; bar represents $50 \mu \mathrm{m}$. means of nonradioactive microspheres and freeze-thawing for visualization. Using this technique hyperglycemia was found to induce a significant reduction of the islet graft's microvascular blood flow, while normalization of islet blood perfusion was encountered after insulin treatment. The decrease of blood flow through the islet grafts was interpreted as result of inadequate revascularization due to the hyperglycemic state of the recipient. In contrast, direct visualization of the microvasculature by means of in vivo microscopy has revealed that hyperglycemia per se does not alter the process of angiogenesis and revascularization of islet isografts transplanted onto striated muscle (7). These findings are in agreement with those of Gray et al. (17), demonstrating that rodent islets implanted beneath the kidney capsule of diabetic rats are not destroyed by two weeks hyperglycemia. The latter authors suggested that protection of islets after implantation by insulin treatment is unnecessary, even in the presence of persistently raised blood glucose levels (17).

In 1989 Jansson and Sandler (8) investigated the influence of hyperglycemia on blood perfusion of autotransplanted islet clusters from rats again by means of microspheres. As reported previously the authors found islet perfusion impaired by hyperglycemia and interpreted this not only as a consequence of inadequate revascularization, but as an intrinsic effect of hyperglycemia on blood flow regulation (8). In contrast, our present study demonstrates that microvascular blood perfusion of re- 

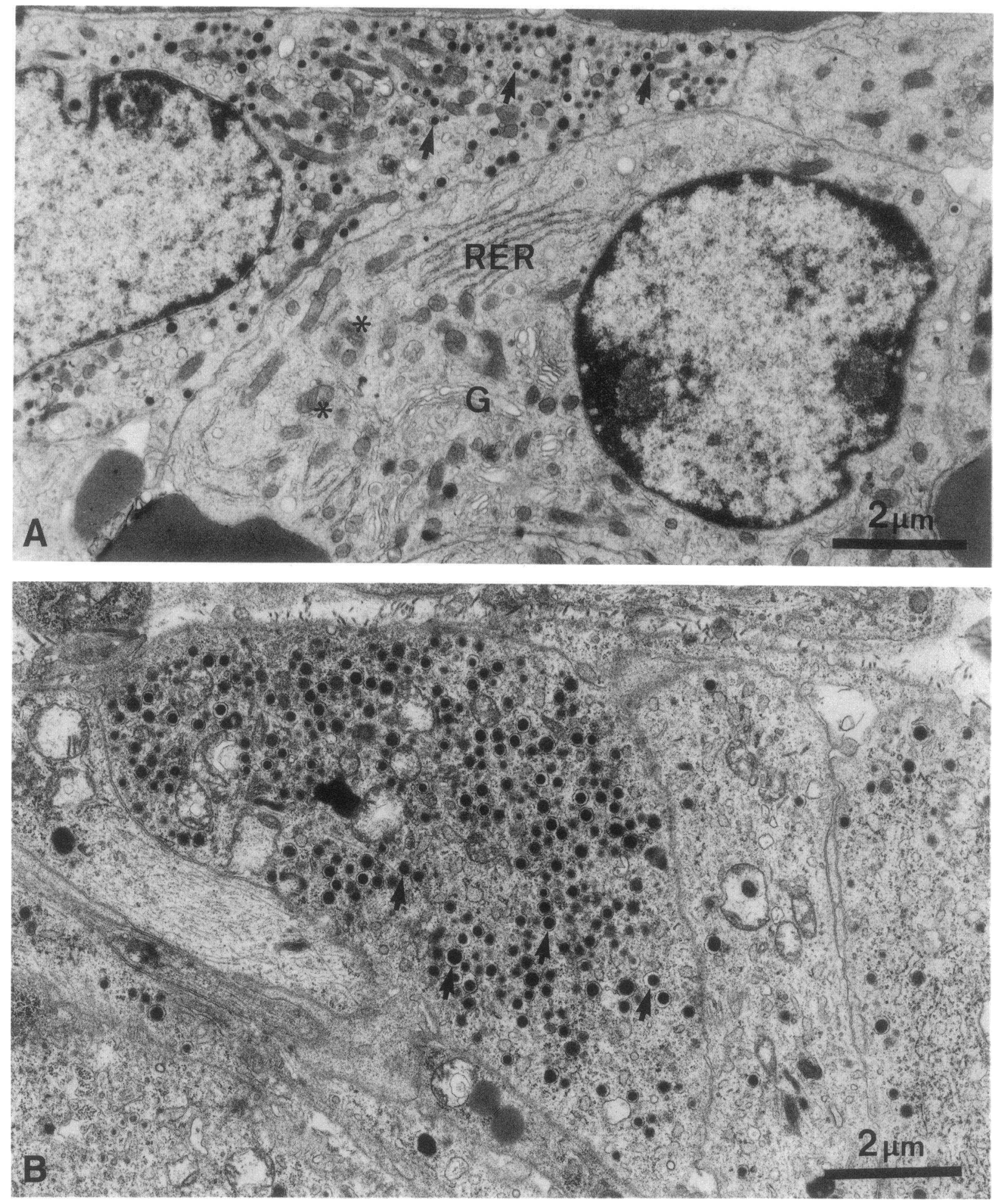

Figure 6. Transmission electron micrographs of pancreatic islet grafts of normoglycemic and hyperglycemic recipients. Transmission electron micrographs of islet isografts 14 days after free transplantation in normoglycemic $(A)$ and hyperglycemic $(B$ and $C)$ recipients. In normoglycemic animals $(A)$ the islet cells revealed normal ultrastructure with their characteristic secretory granules varying in size and electron opacity $(\rightarrow)$. In addition, the cells were characterized by an abundance of rough endoplasmatic reticulum $(R E R)$, Golgi's complex $(G)$, and electron-dense mitochondria $\left({ }^{*}\right)$. Islet cells of hyperglycemic animals also revealed normal ultrastructure $(B$ and $C)$ with augmentation of secretory granules $(\rightarrow, B)$ and rough endoplasmatic reticulum (RER, $C)$, indicating high metabolic activity of these cells, which is also documented by the great number of free ribosomes and polyribosomes $(\triangleright, C)$.

vascularized islet isografts is not affected by hyperglycemia. Islet blood perfusion was found elevated due to the presence of wider capillaries and hence a lower resistance to blood flow.
The islet capillaries did not only show increased diameters, but appeared more tortuous ( Fig. 5), similar as known for the capillaries of pancreatic islets in situ (3). 


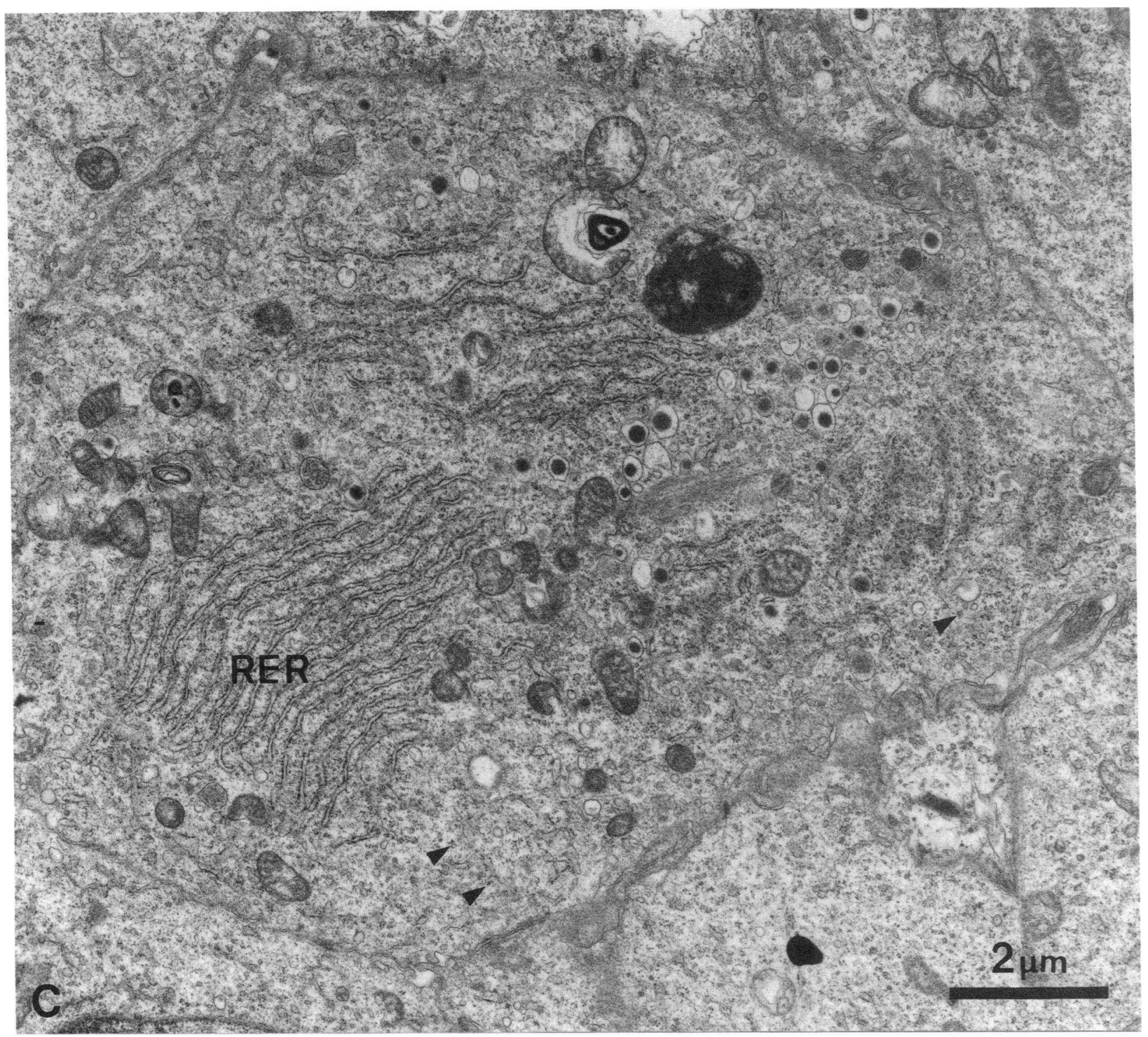

Figure 6 (Continued)

The contradictory results on the influence of hyperglycemia on microvascular blood perfusion of islet grafts may be due to: $(a)$ the procedure applied for islet culture before transplantation; $(b)$ different transplantation modalities, i.e., transplantation of clusters vs. individual islets; $(c)$ the differences of sites (host tissue) chosen for transplantation; and/or $(d)$ the differences in methods used for the assessment of microvascular perfusion.

Culture of isolated islets. Little is known about the influence of $37^{\circ} \mathrm{C}$ culture of isolated pancreatic islets on their potential to induce angiogenesis, revascularization, and microvascular blood perfusion after transplantation. In the hamster, 1 wk culture at $24^{\circ} \mathrm{C}$ and $37^{\circ} \mathrm{C}$, respectively, does not influence the islet graft's potential for angiogenesis and revascularization (18). However, it has been reported that up to $50 \%$ of rat islets are lost in tissue culture or after transplantation within five to seven days after isolation $(19,20)$, and it was hypothesized that islet and/or B cell ischemia may be responsible for functional failure of islet transplants. To exclude influences of islet culture on their potential for revascularization, in the present study islet grafting was performed immediately after the isolation procedure.

Technique for islet transplantation. The aim of this study was to assess capillary blood perfusion of individual islet grafts. Therefore, we have chosen a technique, allowing transplantation of single islets, which thereafter can sequentially be observed by in vivo microscopy (9). In contrast, Sandler and Jansson (6) transplanted islet clusters for analyzing the influence of hyperglycemia on revascularization and microvascular blood perfusion. Isolated islets, whether cultured or immediately transplanted, must be considered to be avascular and hence dependent on diffusion of oxygen and nutrients until revascularization is completed $(21,22)$. While transplantation of single islets onto the striated muscle guarantees direct contact of the islets with the host tissue, transplantation of islet clusters prevents direct contact of most of the islets with the host tissue. Thereby, the diffusion distance for oxygen is increased and hence oxygenation of the islets is impaired; a fact which may explain loss of islet tissue and delayed revascularization as observed after grafting of islet clusters under the renal 
subcapsular space $(21,23)$. In addition, transplantation of single islets onto striated muscle allows for analysis of morphology and microvascular blood perfusion of each individual islet, including its arterial supply, capillary network, and venular drainage system $(9,22)$, whereas transplantation of islet clusters under the kidney capsule prevents assessment of microvascular blood flow of individual islets.

Site of islet transplantation. The site for transplantation may play a major role for successful islet grafting, in particular in regard to the process of angiogenesis and revascularization. Although no ideal site has been identified as yet, some indications of the optimal site are available from experimental studies. It was suggested that the sparsely vascularized subcutaneous and intramuscular site may be associated with absent or poor integration of islet tissue (24), whereas highly vascularized sites such as liver (25), spleen (26), or kidney capsule (27) would allow better functional results.

We have noted after syngeneic transplantation onto striated muscle of Syrian golden hamsters that $97 \%$ of all islets develop a regular microvascular network within a time period of ten days (12), which is in accordance with observations of Griffith et al. (28) based on histological sections from intrahepatically transplanted islet isografts. After portal injection of isolated islets, vascularization of the grafts became visibly apparent at 48 to 72 hours, and by the 11 th day after transplantation revascularization was found completed (28). Since these time ranges are not different from those observed in our skinfold preparation, the process of angiogenesis and revascularization appears not necessarily dependent upon the vascularity of the host tissue.

Sandler and Jansson (6) proposed, that after transplantation of islet clusters beneath the renal capsule revascularization is completed after one month, and for this reason they assessed islet blood perfusion one month after transplantation. However, using intravital microscopy Rooth and coworkers (23) have reported that the process of revascularization of islet clusters autotransplanted beneath the kidney capsule is subjected to a progressive and dynamic modulation of new vessels for a long period, until finally a vascular architecture resembling that of normal pancreatic islets in situ is established at six months. Therefore, cluster transplantation beneath the renal capsule may not represent an adequate model to study revascularization and microvascular blood perfusion of islet grafts.

Technique for the assessment of microvascular blood perfusion of the islet grafts. Evaluation of the microvasculature of free pancreatic islet grafts by means of intravital microscopy allows direct visualization of the microcirculation, including analysis of the microvascular morphology and hemodynamics. High magnification permits quantitative assessment of capillary red blood cell velocity and diameter (see Fig. 4), as well as for analysis of flow behaviour of white blood cells (29). In addition, sequential observations in the awake animal allow for evaluation of dynamic processes, such as angiogenesis and revascularization, without interference by anesthesia $(12,22)$. In contrast, blood flow measurements by means of nonradioactive microspheres using the reference sample technique $(6,8)$ require anesthesia and surgery, and, most importantly, do not allow for analyses of intraindividual changes within the microvasculature.

Furthermore, for the assessment of microvascular blood perfusion of islet grafts the microspheres technique, even when used by the identical investigators, seems to suffer from repro- ducibility, as demonstrated by enormous differences in graft blood flow, despite using the same experimental protocol (see 6 vs. 8). The lack of reproducibility may be due to the fairly low amount of spheres (in some of the clusters only $\sim 25[6]$ ), which were used for measuring blood perfusion of one sample of 500 transplanted islets, and/or due to differences achieved by the calculation of the graft volume (see 6 vs. 8). According to Buckberg et al. (30) the tissue under investigation must contain a minimum of 384 microspheres per sample in order to limit the stochastical error due to the variability of the distribution of the spheres to $5 \%$.

The decrease of blood flow in islet grafts as consequence of hyperglycemia, however, cannot be explained by problems inherent to the variability of distribution when using microspheres, since islet blood flow was found consistently decreased in hyperglycemic recipients $(6,8)$. The low blood flow values measured during hyperglycemia may result from the small diameter $(10.2 \mu \mathrm{m})$ microspheres used. If the capillaries of rat islets transplanted under the kidney capsule would widen to the same extent as the capillaries of hamster islet grafts (present study) approaching diameters of $\sim 11.5 \mu \mathrm{m}$, microspheres with a diameter of $10.2 \mu \mathrm{m}$ would pass the capillary bed and hence falsify the actual flow values.

This phenomenon would also explain the contradictory findings on microvascular blood perfusion in pancreatic islets in situ $(4,5)$ and pancreatic islet autografts $(6,8)$ during hyperglycemia, both evaluated by means of nonradioactive microspheres. Assuming capillary diameters of 5-6 $\mu \mathrm{m}$ in pancreatic islets in situ (31) a hyperglycemia-induced increase of microvascular perfusion due to capillary widening will be indicated by a higher number of trapped $10.2-\mu \mathrm{m}$ microspheres, since capillary diameters will still not exceed the diameter of the microspheres. However, in islet grafts, presenting with capillary diameters of $\sim 9 \mu \mathrm{m}$ (12), a hyperglycemia-induced increase in capillary diameter may exceed the diameter of the microspheres $(10.2 \mu \mathrm{m})$, and therefore, an increase in microvascular perfusion may not be detected, because the spheres will pass the majority of all capillaries.

Vasomotor control of islet graft perfusion during hyperglycemia. Studies on the vasomotor control of pancreatic islets in situ showed that the islet microcirculation is markedly inhibited by sympathomimetic agents, such as epinephrine and ephedrine $(32,33)$, while metacholine chloride, a parasympathomimetic drug, causes vasodilatation and increases microvascular perfusion (33). Hyperglycemia-induced increase of microvascular blood flow of pancreatic islets in situ (2-5) is thought to be mediated either by local and/or nervous and humoral mechanisms. Since glucose stimulates B cell metabolic rate, local thermogenesis (34) and local decrease in blood oxygen saturation (35) might enhance microvascular blood perfusion of pancreatic islets (4). Several lines of evidence suggest that the glucose-sensitive control mechanism is located at an extrapancreatic site. Jansson and Hellerström (36) emphasized the particular role the central nervous system plays in glucose-induced selective increase in islet blood flow. Inasmuch as an increase in islet blood flow due to hyperglycemia was abolished by vagotomy or administration of atropine, the authors hypothesized either cholinergic vasodilatation of the arterioles supplying the islets, or cholinergic release of vasoactive substances (36).

However, the functional response of free pancreatic islet grafts may be considerably different as compared to pancreatic 
islets in situ due to the isolation procedure the grafts have to be subjected to prior to transplantation. Nevertheless, analysis of the morphology of revascularized islet grafts, including the ultrafine structure, revealed a microangioarchitecture similar as compared to pancreatic islets in situ (31) with an intact endothelial lining and B cell structure (22). In addition, the present study demonstrates that individual capillary blood perfusion in islet grafts of normoglycemic recipients is consistently $\sim 1.7$ $\mathrm{nl} / \mathrm{min}$, during the process of revascularization as well as after revascularization was completed, indicating a vasomotor control of capillary perfusion. Capillary blood perfusion is known to vary under different physiological conditions, i.e., at rest and at work, due to changes of demand for oxygen and nutrients. Therefore, the increase of capillary blood perfusion in free pancreatic islet grafts during experimental hyperglycemia might best be interpreted as an adaptation to the increased functional demand of the islet cell under diabetic conditions. We would like to propose that pancreatic islet grafts may have the potential for adequate response during hyperglycemia by increasing microvascular blood perfusion due to humoral and/or vasoactive stimulation, possibly mediated through the central nervous system.

In conclusion, this study has shown that free islet isografts of hamsters transplanted onto striated muscle are revascularized within 10 days after transplantation in normoglycemic and hyperglycemic recipients. Microvascular blood perfusion through islet grafts of hyperglycemic animals is higher as compared to normoglycemic controls, on account of identical capillary density and capillary red blood cell velocity, but increase in capillary diameter and lower microvascular hindrance.

\section{Acknowledgments}

The excellent technical assistance of Beate Wolf is gratefully acknowledged.

This study was supported in part by the Deutsche Forschungsgemeinschaft Me 900/1-1 and Me 900/1-2.

\section{References}

1. Gray, D. W. R., and P. J. Morris. 1987. Developments in isolated pancreatic islet transplantation. Transplantation (Baltimore). 43:321-331.

2. Kracht, J., Y. C. Lo, and J. Rall. 1960. Über Beziehungen zwischen Inselkapillaren und B-Zellfunktion. Endokrinologie. 39:35-43.

3. Hellerstrom, C., and B. Hellman. 1961. The blood circulation of the islets of Langerhans visualized by the fluorescent dye vasoflavine. Acta Soc. Med. Ups. 66:88-94.

4. Jansson, $L$., and C. Hellerström. 1983. Stimulation by glucose of the blood flow to the pancreatic islets of the rat. Diabetologia. 25:45-50.

5. Jansson, L., and S. Sandler. 1985. Pancreatic islet circulation in relation to the diabetogenic action of streptozotocin in the rat. Endocrinology. 116:896-900.

6. Sandler, S., and L. Jansson. 1987. Blood flow measurements in autotransplanted pancreatic islets of the rat. Impairment of the blood perfusion of the graft during hyperglycemia. J. Clin. Invest. 80:17-21.

7. Menger, M. D., S. Jäger, P. Walter, and K. Messmer. 1990. Influence of hyperglycemia on the process of angiogenesis and revascularization of freely transplanted islets of Langerhans. Transplant. Proc. 22:821-822.

8. Jansson, L., and S. Sandler. 1989. Influence of hyperglycemia on blood perfusion of autotransplanted pancreatic islets in diabetic rats. Diabetes. 38(Suppl. 1):196-198.

9. Menger, M. D., S. Jäger, P. Walter, F. Hammersen, and K. Messmer. 1990. A novel technique for studies on the microvasculature of transplanted islets of Langerhans in vivo. Int. J. Microcirc. Clin. Exp. 9:103-117.
10. Lacy, P. E., and M. Kostianovsky. 1967. Method for the isolation of intact islets of Langerhans from the rat pancreas. Diabetes. 16:35-39.

11. Latif, Z. A., J. Noel, and R. Alejandro. 1988. A simple method of staining fresh and cultured islets. Transplantation (Baltimore). 45:827-830.

12. Menger, M. D., S. Jäger, P. Walter, G. Feifel, F. Hammersen, and K. Messmer. 1989. Angiogenesis and hemodynamics of microvasculature of transplanted islets of Langerhans. Diabetes. 38(Suppl. 1):199-201.

13. Endrich, B., K. Asaishi, A. Goetz, and K. Messmer. 1980. Technical report-a new chamber technique for microvascular studies in unanesthetized hamsters. Res. Exp. Med. 177:125-134.

14. Endrich, B., and K. Messmer. 1984. Quantitative analysis of the microcirculation in the awake animal. In Handbook of Microsurgery, Vol. I. W. Olszewski, editor. CRC Press Inc., Boca Raton, FL. pp. 79-105.

15. Baker, M., and H. Wayland. 1974. On-line volume flow rate and velocity profile measurement for blood in microvessels. Microvasc. Res. 7:131-143.

16. Lipowsky, H. H., and B. W. Zweifach. 1978. Technical report. Application of the "two-slit" photometric technique to the measurement of microvascular volumetric flow rates. Microvasc. Res. 15:93-101.

17. Gray, D. W. R., P. McShane, and P. J. Morris. 1986. The effect of hyperglycemia on isolated rodent islets transplanted to the kidney capsule site. Transplantation (Baltimore). 41:699-703.

18. Menger, M. D., S. Jäger, P. Walter, and K. Messmer. 1990. Influence of culture $\left(24^{\circ} / 37^{\circ} \mathrm{C}\right)$ on revascularization of freely transplanted islets of Langerhans. Transplant. Proc. 22:823.

19. Sutherland, D. E. R., J. J. Rynasiewicz, K. Kawahara, P. Gorecki, and J.S Najarian. 1980. Rejection of islets versus immediately vascularized pancreatic allografts: a quantitative comparison. J. Surg. Res. 29:240-247.

20. Selawry, H., M. M. Mui, R. D. Paul, and J. A. Distasio. 1984. Improved allograft survival using highly enriched populations of rat islets. Transplantation (Baltimore). 37:202-205.

21. Stagner, J. I., and E. Samols. 1990. The induction of capillary bed development by endothelial cell growth factor before islet transplantation may prevent islet ischemia. Transplant. Proc. 22:824-828.

22. Menger, M. D., and K. Messmer. 1991. The microvasculature of free pancreatic islet grafts. In Pathways to Applied Immunology. K. Messmer and M. Stein, editors. Springer-Verlag, Berlin. pp. 109-126.

23. Rooth, P., I. Dawidson, K. Lafferty, K. Diller, J. Armstrong, P. Pratt, R. Simonsen, and I.-B. Täljedal. 1989. Prevention of detrimental effect of cyclosporin $A$ on vascular ingrowth of transplanted pancreatic islets with verapamil. Diabetes. 38(Suppl. 1):202-205.

24. Gray, B. N., and E. Watkins. 1976. Isolated islet transplantation in experimental diabetes. Aust. J. Exp. Biol. Med. Sci. 54:57-60.

25. Kemp, C. B., M. J. Knight, D. W. Scharp, W. F. Ballinger, and P. E. Lacy. 1973. Effect of transplantation site on the results of pancreatic islet isografts in diabetic rats. Diabetologia. 9:486-491.

26. Finch, D. R., P. H. Wise, and P. J. Morris. 1977. Successful intra-splenic transplantation of syngeneic and allogeneic isolated pancreatic islets. Diabetologia. 13:195-199.

27. Reece-Smith, H., D. F. DuToit, P. McShane, and P. J. Morris. 1981. Prolonged survival of pancreatic islet allografts transplanted beneath the renal capsule. Transplantation (Baltimore). 31:305-306.

28. Griffith, R. C., D. W. Scharp, B. K. Hartman, W. F. Ballinger, and P. E. Lacy. 1977. A morphologic study of intrahepatic portal-vein islet isografts. Diabetes. 26:201-214.

29. Menger, M. D., S. Jäger, P. Walter, F. Hammersen, and K. Messmer. 1991. Microvascular phenomena during pancreatic islet graft rejection. Langenbecks Arch. Chir. 376:214-221

30. Buckberg, G. D., J. C. Luck, D. B. Payne, J. I. E. Hoffman, J. P. Archie, and D. E. Fixler. 1971. Some sources of error in measuring regional blood flow with radioactive microspheres. J. Appl. Physiol. 31:598-604.

31. Menger, M. D., F. Hammersen, and K. Messmer. 1990. The microcirculation of the islets of Langerhans: present state of the art. Prog. Appl. Microcirc. 17:192-215.

32. Rooth, P., and I.-B. Täljedal. 1987. Vital microscopy of islet blood flow: catecholamine effects in normal and ob/ob mice. Am. J. Physiol. 252:E130E135.

33. Bunnag, S. C., N. E. Warner, and S. Bunnag. 1977. Vasomotor reactions in the islets affecting the blood glucose levels. Bibl. Anat. 16:445-449.

34. Gylfe, E., and B. Hellman. 1975. The heat production of pancreatic B-cells stimulated by glucose. Acta Physiol. Scand. 93:179-183.

35. Hellerström, C. 1967. Effects of carbohydrates on the oxygen consumption of isolated pancreatic islets of mice. Endocrinology. 81:105-112.

36. Jansson, L., and C. Hellerström. 1986. Glucose-induced changes in pancreatic islet blood flow mediated by central nervous system. Am. J. Physiol. 251:E644-E647. 